is of importance alike academically from the point of view of fundamental research, and practically and economically from the point of view of preserving the fisheries and permitting harmless waste effluent. Actual research work on the subject involves, however, a large amount of correlated biological, hydrographical, and chemical field work supplemented by work in laboratories, and requires the collaboration of specialists in those branches of inquiry. The main problem is, however, clearly a biological one.

It is submitted that regular work on the general conditions of both unpolluted and polluted local streams, rivers, and estuaries should be begun at once with the view of detecting or disproving pollution effects, and of building up a body of information on which can be founded practical observations to determine ultimately what degree of dilution should be brought about to make waste effluents harmless, since the prevention of pollution is the aim of all.

It is thus clear that financial support for researches of the kind noted above can be claimed equally from these bodies interested in fostering fundamental researches of any kind, from those manufacturers interested in the use of rivers for waste effluents, as well as from those interested in the preservation of healthy fishing runs, such as County Councils, local Fishery Boards, and Angling Societies.

\section{J. H. ORTON.}

Plymouth and Exeter, January $\mathbf{1 6}$.

\section{The Vector Quantum.}

THE shift, recently discovered by A. H. Compton, in the spectral lines due to the scattering process, or rather the explanation of the shift proposed (Phys. Rev. p. 483 , vol. 2 I, No. 5, May I923) by Compton on the basis of the scattering of one quantum by one electron, furnishes considerable support for a corpuscular theory of radiation. On the basis of a corpuscular theory, an effect, recently discovered by the present writer (NATURE, September 8, I923) takes on a new significance. The effect was discovered by taking photographs by Wilson's cloud expansion method, of the tracks of photo-electrons ejected by plane polarised X-rays. Such photographs bring out the fact that most of the photo-electrons are ejected nearly parallel to the electric force of the radiation. Variations in direction of ejection on all sides of the electric force may be accounted for by the initial momentum of the electron in its atomic orbit.

Now the " electric force" of a polarised radiation is a term deriving most of its meaning from the classical wave theory. On a corpuscular theory, why should the quantum eject the photo-electron sideways out of the atom? Evidently, if we are to conceive of the quantum of radiant energy as a corpuscle of forward momentum $h \nu / c$, as Compton's theory would seem to indicate, we are obliged to conclude that such a corpuscle is not a mere scalar bundle of energy, but possesses a sideways vector property. For, in delivering its energy to the photo-electron, the quantum explodes, so to speak, at right angles to its direction of motion and in a definite plane. Furthermore, from polarisation phenomena, we conclude that such a "vector quantum" once started on its way maintains its vectorial properties constant in direction. Thus we arrive at a conception of the vector quantum as a corpuscle of energy proceeding with the velocity of light, having a forward momentum $h v / c$ and possessing the vector property, which it maintains constant in direction as it proceeds, of imparting its energy by a sideways impulse.

$$
\text { NO, } 2833 \text {, VOL. I I } 3 \text { ] }
$$

On such a view we should naturally look for some evidence of a reaction to the sideways impulse which the vector quantum exerts upon the photo-electron. With a concentrated vector bundle of energy torn from its source and out in space alone we could scarcely expect the ether or the source of the quantum to be a likely seat for the reaction to exert itself upon. This seems to leave us with the atomic nucleus or another electron as the only seat for the reaction impulse.

If another electron acts as the seat to the reaction impulse, it will receive a momentum equal and opposite to that of the photo-electron and about the same energy as the photo-electron. We should then get two photo-electrons having atuut the same range and starting near the same point. Rather striking confirmation of this view is given by some photographs recently published (Proc. Roy. Soc., fig. 22, plate I2, vol. I04, August 1923) by C. T. R. Wilson. This and other photographs show strange pairs of associated tracks having about the same range and starting near the same point. This confirmation is rendered particularly striking by a remark of Wilson's, " There is, as with pairs of the preceding class, a great tendency for the line joining the points of origins of the two members of a pair to be nearly perpendicular to the primary X-ray beam."

If, on the other hand, the atomic nucleus suffers the reaction impulse, it will receive an equal and opposite momentum to that of the photo-electron, but, since its mass is of the order of 2000 times that of the photo-electron, its energy from the vector quantum would only be of the order of $1 / 2000$ of that of the photo-electron. This should require the introduction of a small term into Einstein's photo-electric equation to take account of the kinetic energy given to the nucleus.

Washington University, Saint Louis, December 27.

\section{Rapid Variations of the Earth's Potential Gradient.}

In the course of experiments on atmospherics carried out for the Radio Research Board, it has been found that many of these disturbances include a semipermanent net change of the earth's potential gradient of the order of $\mathrm{O} \cdot \mathrm{I}$ volt per metre. It is natural to consider this change of field as representing the electrostatic effect of the thundercloud, the discharge of which causes an atmospheric. Measurements of the sign and magnitude of this change of field have been made by observing the alteration of the total electric charge on an exposed aerial system of high capacity (cf. C. T. R. Wilson, Proc. Roy. Soc. A, vol. 92 , p. 555 , r916). It is estimated that these observations were made at least 200 kilometres from the thundercloud in question, in which case we may be fairly certain that the sign of the electric field change is indicative of the sign of the total electric moment destroyed in the discharge. It is found that the field changes in question are predominantly of one sign, representing the destruction of a field directed towards the earth. The ratio of the number of field changes of this sign to those of the opposite sign so far observed is about 300 to $\mathbf{I}$.

It thus must be concluded that, whatever the disposition of the positive and negative charges dissipated in the discharge, the resultant electric moment of these charges with respect to the earth is predominantly positive.

It might at first sight appear that these results are not in agreement with Mr. C. T. R. Wilson's measurements of the electrostatic field changes made at short 